\title{
Ursodeoxycholic Acid Triggers Primary Enterolith Growth in a Crohn's Disease Patient with Jejunal Stenosis
}

\author{
Hiroki Matsui ${ }^{1)}$, Tadashi Yoshida ${ }^{1)}$, Shigenori Homma ${ }^{1)}$, Nobuki Ichikawa ${ }^{1)}$, Shin Emoto ${ }^{1)}$, Yoichi Miyaoka ${ }^{1)}$ \\ Kensuke Sakurai ${ }^{2)}$, Shinsuke Odagiri ${ }^{2}$, Takehiko Katsurada ${ }^{2)}$ and Akinobu Taketomi ${ }^{1)}$ \\ 1) Department of Gastroenterological Surgery I, Hokkaido University Graduate School of Medicine, Sapporo, Japan \\ 2) Department of Gastroenterology, Hokkaido University Graduate School of Medicine, Sapporo, Japan
}

\begin{abstract}
Primary enteroliths associated with Crohn's disease have been considered to be rare and are most likely caused by severe ileal stenosis. Herein, we report the case of a primary enterolith possibly caused by mild jejunal stenosis in a Crohn's disease patient who received oral administration of ursodeoxycholic acid (UDCA). A 62-year-old woman with a 6-year history of Crohn's disease, currently in clinical remission, was on UDCA prescription for liver dysfunction. Magnetic resonance imaging and double-balloon endoscopy, which were performed to examine epigastric pain, revealed mild jejunal stenosis and an enterolith on the oral side. Since it was difficult to remove or crush the enterolith endoscopically, we decided to remove it surgically with the stenotic jejunum. Component analysis revealed that more than $98 \%$ of the enterolith was composed of UDCA; subsequently, oral administration of UDCA was discontinued. This case demonstrated that primary enterolith might develop in Crohn's disease patients with mild intestinal stenosis, and oral administration of UDCA can trigger an enterolith in such patients. Therefore, routine follow-up imaging is necessary for early detection. Oral UDCA should be administered with caution for Crohn's disease patients with stenosis of the proximal small intestine.
\end{abstract}

\section{Keywords}

Crohn's disease, primary enterolith, stenosis of proximal small intestine, ursodeoxycholic acid

J Anus Rectum Colon 2021; 5(4): 433-438

\section{Introduction}

Stones in the gastrointestinal tract, or enteroliths, can cause intestinal obstruction, erosion, ulcers, bleeding, and perforations and, therefore, require therapeutic interventions, including surgery[1]. Enteroliths are often classified into primary enteroliths that develop in the intestinal tract and secondary enteroliths that originate outside the intestinal tract and move into the intestinal tract through the fistulas[1]. Primary enteroliths develop in areas of intestinal stasis, and one of the causes of intestinal stasis is intestinal stenosis[1]. Crohn's disease, which has been identified as the cause of intestinal stenosis, has been determined to cause primary enteroliths $[1,2]$. However, reports of primary enteroliths associated with Crohn's disease remain limited, and most reported enteroliths are determined to be caused by severe ileal stenosis after a long period of illness[2-5]. To the best of our knowledge, no studies have so far explored the relationship between primary enteroliths associated with Crohn's disease and the oral administration of ursodeoxycholic acid (UDCA). Herein, we report the case of a patient with primary enterolith possibly caused by mild jejunal stenosis due to Crohn's disease and oral administration of UDCA. 

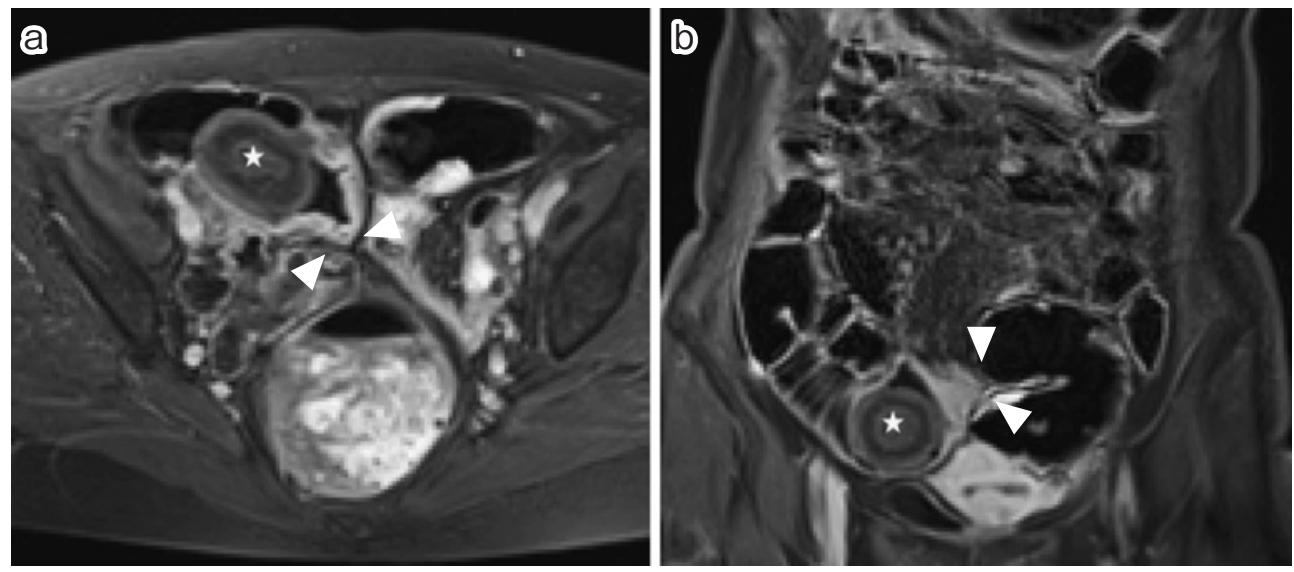

Figure 1. Magnetic resonance imaging (MRI) findings.

a) Transverse plane. b) Frontal plane. MRI shows a $4.5-\mathrm{cm}$ oval structure in the small intestine in the pelvis (white star). A stenosis of the small intestine was also detected via MRI with the thickening of the intestinal wall on the anal side of the oval structure (white arrowhead); however, there is no evidence of intestinal obstruction. There are no other abnormal findings on MRI.

\section{Case Report}

A 62-year-old woman with Crohn's disease underwent magnetic resonance imaging (MRI) for transient epigastric pain and was then diagnosed with an enterolith. She had no medical history of biliary tract disease, intestinal diverticulosis, or urinary tract disease. About 6 years ago, she had been examined for upper abdominal pain and was diagnosed with Crohn's disease. She was in remission after receiving prednisolone (PSL), azathioprine (AZA), and infliximab (IFX). After induction of remission, PSL was discontinued, and the administration of AZA and IFX maintained her clinical remission. She was later diagnosed with hepatic dysfunction 5 years ago and received a UDCA prescription $(600 \mathrm{mg} / \mathrm{day})$. Double-balloon enteroscopy (DBE) 2 years prior did not show enteroliths or significant intestinal stenosis. An MRI performed 1 month before surgery due to transient epigastric pain examination revealed a $4.5-\mathrm{cm}$-sized oval structure in the small intestine (Figure 1), which was later diagnosed as an enterolith. The MRI and additional computed tomography (CT) showed stenosis of the small intestine with the thickening of the intestinal wall just on the anal side of the enterolith without any evidence of intestinal obstruction (Figure 1). The imaging did not show any abnormal findings, including in the biliary system or urethral system. Transanal DBE showed a stenotic site in the jejunum, located $250 \mathrm{~cm}$ from the terminal ileum (Figure 2a). The jejunal stenosis was mild enough for DBE to pass, with DBE revealing the presence of an enterolith on the oral side of the stenosis (Figure 2b). We failed to remove and crush the enterolith endoscopically (Figure 2b). Therefore, we decided to surgically remove the enterolith. Ports were inserted into the umbilical region, left abdomen, and lower left abdomen to initi- ate the laparoscopic procedure. The dilated jejunum with the enterolith was found without any adhesions. We then extended the umbilical incision to the cranial and caudal sides and guided the intestinal tract, which was grasped with forceps, to the exterior of the incision. The stenotic site was located in the jejunum, approximately $150 \mathrm{~cm}$ from the Treitz ligament, along with the enterolith (Figure 3). No other stenosis or strictures were detected in other parts of the small intestine. Because the enterolith was large and its removal required a large intestinal incision, we were concerned that this intervention would complicate the subsequent procedure. Furthermore, even if we performed a small bowel resection, we thought that the remaining small bowel would be sufficient. Therefore, the jejunum, including the stricture, was resected along with the enterolith. The jejunum was reconstructed using functional end-to-end anastomosis. The surgery duration was 1 hour and 32 minutes, and the amount of bleeding was insignificant. She was later discharged without complications on the 13th day after the surgery. The stenotic site of the resected jejunum showed inflammation and fibrous changes without malignant findings (Figure 4). The enterolith appeared yellowish-brown and oval with a diameter of $4.5 \mathrm{~cm}$ (Figure $5 \mathrm{a}$ ). The cut surface was yellow and had a multilayered structure (Figure 5b). As per the component analysis, it was determined that more than $98 \%$ of the enterolith was composed of UDCA. Subsequently, oral administration of UDCA was discontinued after surgery (Figure 5c).

\section{Discussion}

This case report highlights two important clinical issues. First, this case showed that Crohn's disease patients may de- 

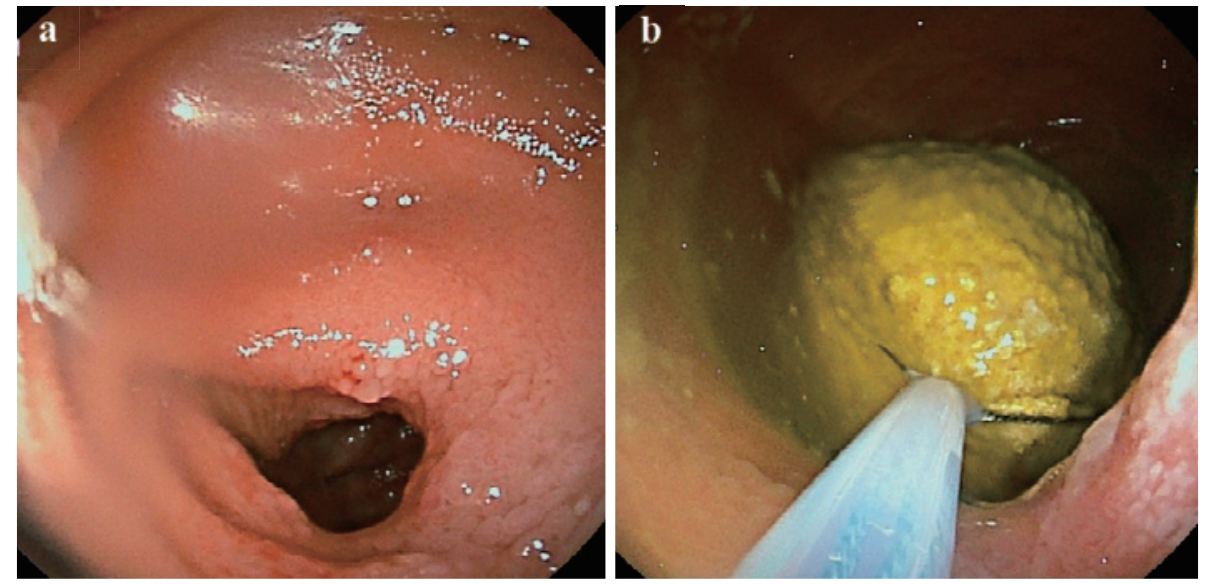

Figure 2. Transanal double-balloon enteroscopy (DBE) findings.

a) Transanal DBE revealed mild stenosis with an ulcer in the jejunum, approximately 250 $\mathrm{cm}$ proximal to the terminal ileum. b) The enteroscope that was inserted through the stenotic site of the jejunum revealed a yellowish-brown oval enterolith on the oral side. Attempts to remove and crush the enterolith using biopsy forceps and snare forceps were unsuccessful. Removal of the enterolith by oral DBE was considered impossible because its large size made it difficult to grasp it securely with snare forceps and to pass it through the pylorus.

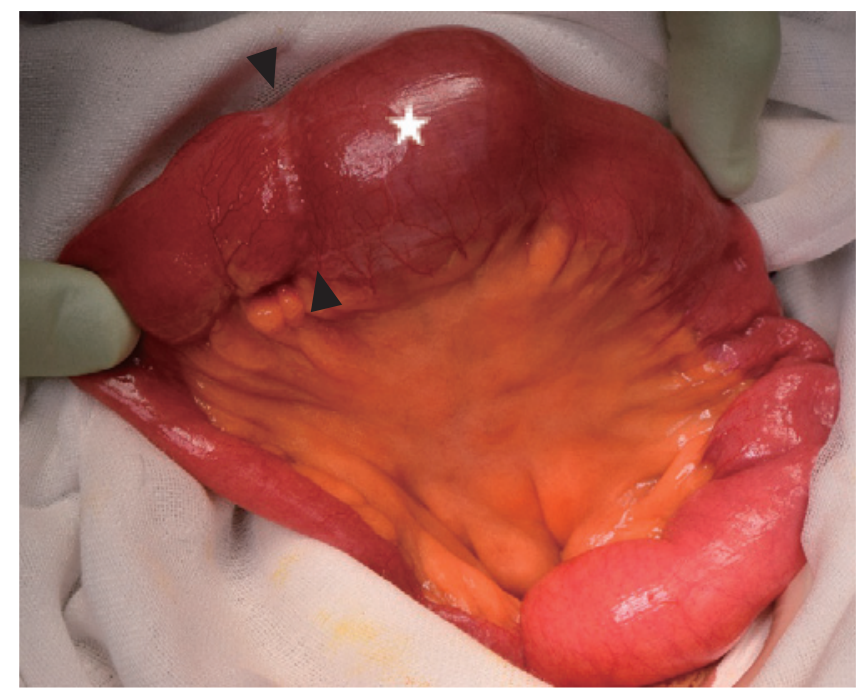

Figure 3. Intraoperative findings.

A stenotic site with thickening of the intestinal wall was found in the jejunum, approximately $150 \mathrm{~cm}$ distal to the Treitz ligament (black arrowhead). The hard oval enterolith on the oral side is guided to the stenotic site of the jejunum but cannot be moved to the anal side of the stenotic site (white star).

velop a primary enterolith due to mild intestinal stenosis. As mentioned earlier, there are two types of enteroliths, that is, primary enteroliths that develop in the intestinal tract and secondary enteroliths that originate outside the intestinal tract and move into the intestinal tract through the fistulas[1]. Most secondary enteroliths develop in the biliary system and the urinary system[1,6]. Our patient was diagnosed with primary enterolith because of the absence of medical history or imaging abnormality in the biliary or urinary systems. Intestinal stenosis can often lead to stasis, which may further cause primary enteroliths[1]. Thus, intestinal stenosis associated with Crohn's disease can be considered as a risk factor for primary enteroliths[1,2]. Mendes et al. reviewed 22 cases of enteroliths caused by Crohn's disease, wherein it was found that enteroliths associated with Crohn's disease developed on average 15.7 years after disease onset, and they were associated with severe intestinal stenosis formed after a long illness period[2]. However, in our case, the enterolith formed shortly after the diagnosis of Crohn's disease, about 6 years after onset, due to mild intestinal stenosis that was penetrable by DBE. Enteroliths that develop under mild intestinal stenosis can be expected to be excreted in stools and are unlikely to cause clinical problems. However, in our patient, the enterolith could not pass through the stenotic site of the intestine even if it was only a case of mild intestinal stenosis. Fortunately, our patient showed only minor symptoms, and the enterolith did not cause any clinical problems, but such enteroliths can cause intestinal obstruction, erosions, ulcers, bleeding, and perforation, and their early detection is important. In this case, the enterolith appears to have developed in less than 2 years, since a DBE performed 2 years prior did not indicate enteroliths or significant intestinal stenosis. Therefore, we propose to followup on patients with Crohn's disease every 1-2 years using CT or MRI, which have high detection rates for enteroliths[2,4,7].

Second, this case showed that the oral administration of UDCA can trigger the formation of enteroliths in Crohn's disease patients with jejunal stenosis. Primary enteroliths in- 

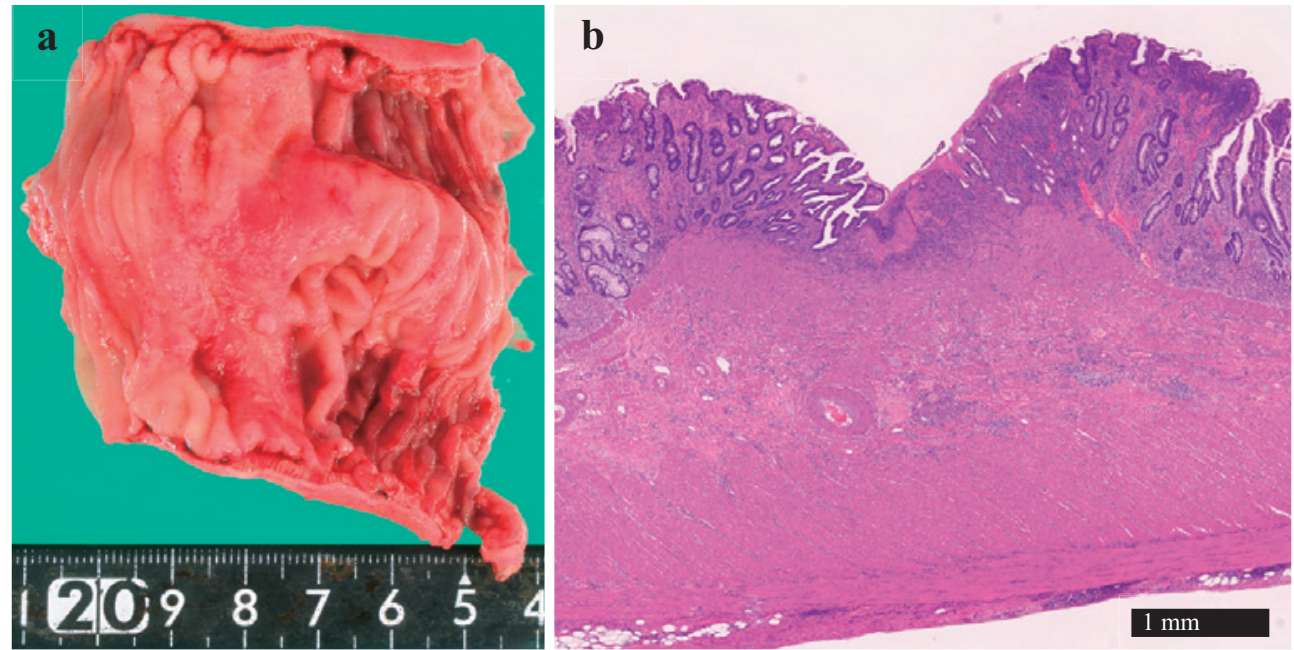

Figure 4. Findings of the resected specimen.

a) Inflammatory changes in the intestinal mucosa, scarring, and thickening of the intestinal wall were macroscopically observed at the stenotic site of the jejunum, but no neoplastic lesions were detected. b) Histologically, a partial erosion was observed at the stenotic site of the jejunum, accompanied by the thickening of the mucosa and muscularis mucosae and fibrosis of the submucosa. There were no histological findings indicating malignancy.

clude bile acid-based enteroliths that are known to develop under the acidic environment of the proximal small intestine and calcium-based enteroliths that develop under the alkaline environment of the distal small intestine or large intestine $[1,8]$. Crohn's disease causes intestinal stenosis in all parts of the gastrointestinal tract. Among them, intestinal stenosis frequently occurs in the ileum[2,3]. In addition, Crohn's disease patients are determined to often have a reduced bile acid pool due to impaired bile acid absorption at the terminal ileum[9,10]. Therefore, most enteroliths associated with Crohn's disease are considered to be calciumbased enteroliths in the distal small intestine. Previous reports have shown that enteroliths associated with Crohn's disease are predominantly calcium-based enteroliths in the ileum[2,4,7]. However, in our patient, the enterolith was bile acid-based and developed in the jejunum; it was suspected to be due to jejunal stenosis and oral administration of UDCA $(600 \mathrm{mg} / \mathrm{day}$ for the past 5 years $)$. We believe that orally administered UDCA might have precipitated to form the enterolith either directly or after enterohepatic circulation in intestinal stasis under the acidic environment caused by the jejunal stenosis. This theory was supported by the results of component analysis, which showed that more than $98 \%$ of the enterolith was composed of UDCA.

In this case, we consider that the enterolith developed when intestinal stasis in an acidic environment due to mild jejunal stenosis was combined with oral administration of UDCA. Oral administration of UDCA was started 5 years ago, but the DBE 2 years ago revealed neither jejunal stenosis nor enteroliths. It is likely that mild jejunal stenosis oc- curred during the last 2 years and, combined with oral administration of UDCA, caused the enterolith formation.

To the best of our knowledge, there are no reports of enteroliths caused by the oral administration of UDCA in Crohn's disease patients. However, some case reports have described enteroliths that developed during the oral administration of UDCA in patients who underwent hepaticojejunostomy during living-donor liver transplantation or in those who underwent Billroth II anastomosis when gastrectomy was performed for gastric ulcer. In both cases, the authors consider that the reconstruction caused intestinal stasis under the acidic environment in the elevated jejunum, resulting in the development of enteroliths[11,12]. In another case, four patients had recurring bile duct stones that developed during oral administration of UDCA. One of the four patients underwent choledochoduodenostomy, while one underwent hepaticoduodenostomy. A component analysis of resected samples revealed UDCA as the main component of the stones, and bile duct stones did not develop after discontinuing UDCA[13]. It is possible that, in these patients, the reconstruction caused stasis under the acidic environment in the common bile duct or hepatic duct, resulting in UDCA precipitation leading to the formation of bile duct stones. The oral administration of UDCA can trigger the formation of enteroliths not only in jejunal stenosis patients due to Crohn's disease but also in pathological conditions that can cause stasis under an acidic environment. Oral UDCA should be administered cautiously in such patients.

In conclusion, this case report has demonstrated that in Crohn's disease patients, a primary enterolith may develop 

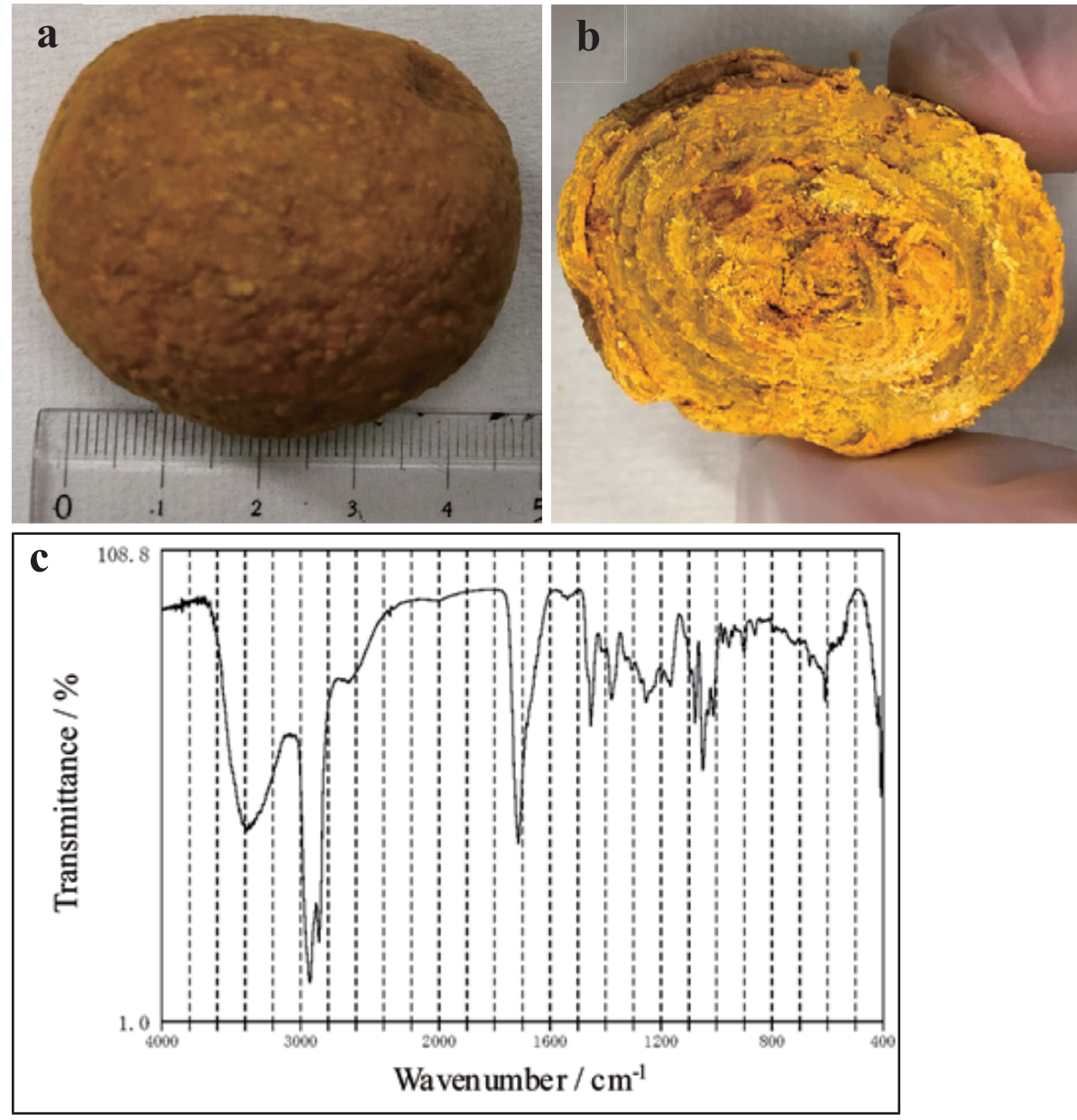

Figure 5. Enterolith findings.

a) The enterolith, yellowish-brown in color, oval-shaped, and hard, with a maximum diameter of $4.5 \mathrm{~cm}$. b) The cut surface is yellow and has a multilayered structure. c) Component analysis by infrared spectroscopy revealed that more than $98 \%$ of the enterolith is composed of ursodeoxycholic acid.

as a result of mild intestinal stenosis after a relatively short period of illness, and oral administration of UDCA could trigger the development of an enterolith in a Crohn's disease patient with jejunal stenosis. Primary enteroliths may develop even in asymptomatic Crohn's disease patients, and follow-up with CT or MRI is necessary for the early detection of enteroliths that may cause complications. In addition, the oral administration of UDCA should be considered with caution not only for Crohn's disease patients with proximal intestinal stenosis but also in cases where intestinal stasis may occur under an acidic environment.

Conflicts of Interest

There are no conflicts of interest.

\section{Author Contributions}

H.M. and S.H. contributed to the conception of the presented idea. T.Y., S.H., and S.E. performed the operation. H.
M. wrote the manuscript with support from T.Y. All authors discussed the text content and contributed to the final manuscript. A.T. gave the final approval for the version to be published.

Approval by Institutional Review Board (IRB)

This study did not require IRB approval because of case report without medical intervention summarizing not more than nine cases.

\section{Informed Consent}

The patient provided written informed consent for publication of this case report.

\section{References}

1. Gurvits GE, Lan G. Enterolithiasis. World J Gastroenterol. 2014 Dec; 20(47): 17819-29.

2. Ribeiro HKM, Nolan DJ. Enterolithiasis in Crohn's disease. Ab- 
dom Imaging. 2000 Oct; 25(5): 526-9.

3. Perathoner A, Kogler P, Denecke C, et al. Enterolithiasisassociated ileus in Crohn's disease. World J Gastroenterol. 2012 Nov; 18(42): 6160-3.

4. Tewari A, Weiden J, Johnson JO. Small-bowel obstruction associated with Crohn's enterolith. Emerg Radiol. 2013 Aug; 20(4): 3414.

5. Jones MW, Koper B, Weatherhead WF. Crohn's disease with enterolith treated laparoscopically. JSLS. 2005 Jul-Sep; 9(3): 339-41.

6. Lassandro F, Romano S, Ragozzino A, et al. Role of helical CT in diagnosis of gallstone ileus and related conditions. Am J Roentgenol. 2005 Nov; 185(5): 1159-65.

7. Wide JM, Loughran CF, Shoker BS. Crohn's disease, calculi and cancer: a report of two cases. Clin Radiol. 1996 Sep; 51(9): 651-3.

8. Quazi MR, Mukhopadhyay M, Mallick NR, et al. Enterolith containing uric acid: an unusual cause of intestinal obstruction. Indian J Surg. 2011 Aug; 73(4): 295-7.

9. Vantrappen G, Ghoos Y, Rutgeerts P, et al. Bile acid studies in un- complicated Crohn's disease. Gut. 1977 Sep; 18(9): 730-5.

10. Rutgeerts P, Ghoos Y, Vantrappen G. Bile acid studies in patients with Crohn's disease. Gut. 1979 Dec; 20(12): 1072-7.

11. Mukai S, Onoe T, Tashiro H, et al. Small bowel obstruction due to an unconjugated ursodeoxycholic acid enterolith following living donor liver transplantation: report of a case. Hepatol Res. 2015 Jul; 45(7): 818-22.

12. Inoguchi K, Yoshioka $\mathrm{T}$, Gomi S, et al. A case of enterolith associated with perforation of jejunal diverticula in the afferent loop. Jpn J Gastroenterol Surg. 2003 Nov; 36(11): 1575-80 (in Japanese).

13. Akiyama $S$, Imamura $T$, Tamura $T$, et al. Recurrent common bile duct stones composed of ursodeoxycholic acid: a report of four cases. Intern Med. Nov. 2014; 53(21): 2489-92.

Journal of the Anus, Rectum and Colon is an Open Access journal distributed under the Creative Commons Attribution-NonCommercial-NoDerivatives 4.0 International License. To view the details of this license, please visit (https://creativ ecommons.org/licenses/by-nc-nd/4.0/). 